Pathologe 2020 • 41:3-13

https://doi.org/10.1007/s00292-019-00732-4

Online publiziert: 13. Januar 2020

(c) Der/die Autor(en) 2019

Schwerpunktherausgeber

R. Büttner, Köln

S. Schäfer, Köln

Britta Janina Wagner · Reinhard Buettner

Institut für Pathologie, Universitätsklinikum und Centrum für Integrierte Onkologie (CIO) Köln, Köln Deutschland

\title{
Immunhistochemische und molekularpathologische Diagnostik von Lungenkarzinomen
}

und atypischen Karzinoiden und großzelligen NEK. Gleichzeitig sind wie bei kaum einem anderen soliden Karzinomtyp zahlreiche molekulare Treibermutationen und immuntherapeutisch relevante Signalmoleküle entdeckt worden, sodass die praktische Diagnostik eine rasche, umfassende und sensitive molekularpathologische Diagnostik erfordert [5, 23]. Zugleich hat ein rascher technologischer Fortschritt, wie z. B. endobronchiale Navigationstechnologien und bildgebungsgesteuerte Nadelaspirationstechniken, zu einem dramatischen Wandel der Biopsiematerialien geführt. Heute dominieren sehr kleine Aspirationsbiopsien mit systematischem Tumorund Lymphknotenmapping das histopathologische Einsendegut. Diese Technologien liefern Minihistologien oder zytologische Materialien in höchster diagnostischer Qualität und erfordern bei der Komplexität der notwendigen Diagnostik durch den Pathologen ein strategisches Vorgehen, um unnötigen Materialverlust zu vermeiden [13]. Die Anforderungen an den Pathologen sind exponentiell gewachsen und zugleich die diagnostischen Materialmengen auf ein Minimum geschrumpft.

Wir schlagen daher ein diagnostisches 3-Stufen-Schema vor, das beginnend mit der Aufarbeitung im Labor zum einen alle weiteren Schritte im Blick hat und zum anderen auf der Verfügbarkeit von histologischen Materialien (EBUS-TBNA, EBB, TBB, CT-gesteuerten Nadelbiopsien etc.) oder von eingebetteten Zytologien („Zytoblock“ [16]) beruht (• Abb. 1).
Im 1. Schritt werden 2 histologische Schnitte $(1 \times$ HE und $1 \times$ PAS) angefertigt. Diese führen zur Arbeitsdiagnose (NSCLC, SCLC, LCNEC, „salivary gland-like carcinoma“, andere Histologien wie Lymphom, Sarkom, Mesotheliom).

Im 2. Schritt werden IHC-Färbungen in Richtung der Differenzierung verschiedener Lungentumoren angeschlossen. Lautet die Arbeitshypothese nicht NSCLC oder wird klinisch nach einer Metastase eines bekanntem Primärkarzinom gefragt, wird eine entsprechende IHC eingeleitet und eine finale Diagnose erstellt (s. Abschn. „Immunhistochemische Diagnostik").

Falls die Arbeitshypothese NSCLC lautet, erfolgen IHC-Färbungen für p40 und TTF1. Bei Verdacht auf SCLC empfehlen wir eine Färbung mit Synaptophysin und CD56, wobei CD56 immer in Kombination mit Ki67 (MIB1) gefärbt werden sollte. Bei Verdacht auf ein Karzinoid sollte eine Chromogranin-A-Färbung erfolgen. Für die molekularpathologische Diagnostik für Adenokarzinome oder Plattenepithelkarzinome werden mit einem wasserfesten Stift Tumorareale markiert und der Prozentsatz des Tumorzellgehalts mikroskopisch für die Makrodissektion abgeschätzt. Im molekulardiagnostischen Labor werden vom Block unter den Kautelen der Kontaminationsvermeidung (Messerwechsel im Mikrotom nach jedem Gewebeblock, Wechsel des Wassers im Wasserbad) Schnitte für IHC-Färbungen, für FISHUntersuchungen und für die DNA-Exmaßen für NET einschließlich typischen 


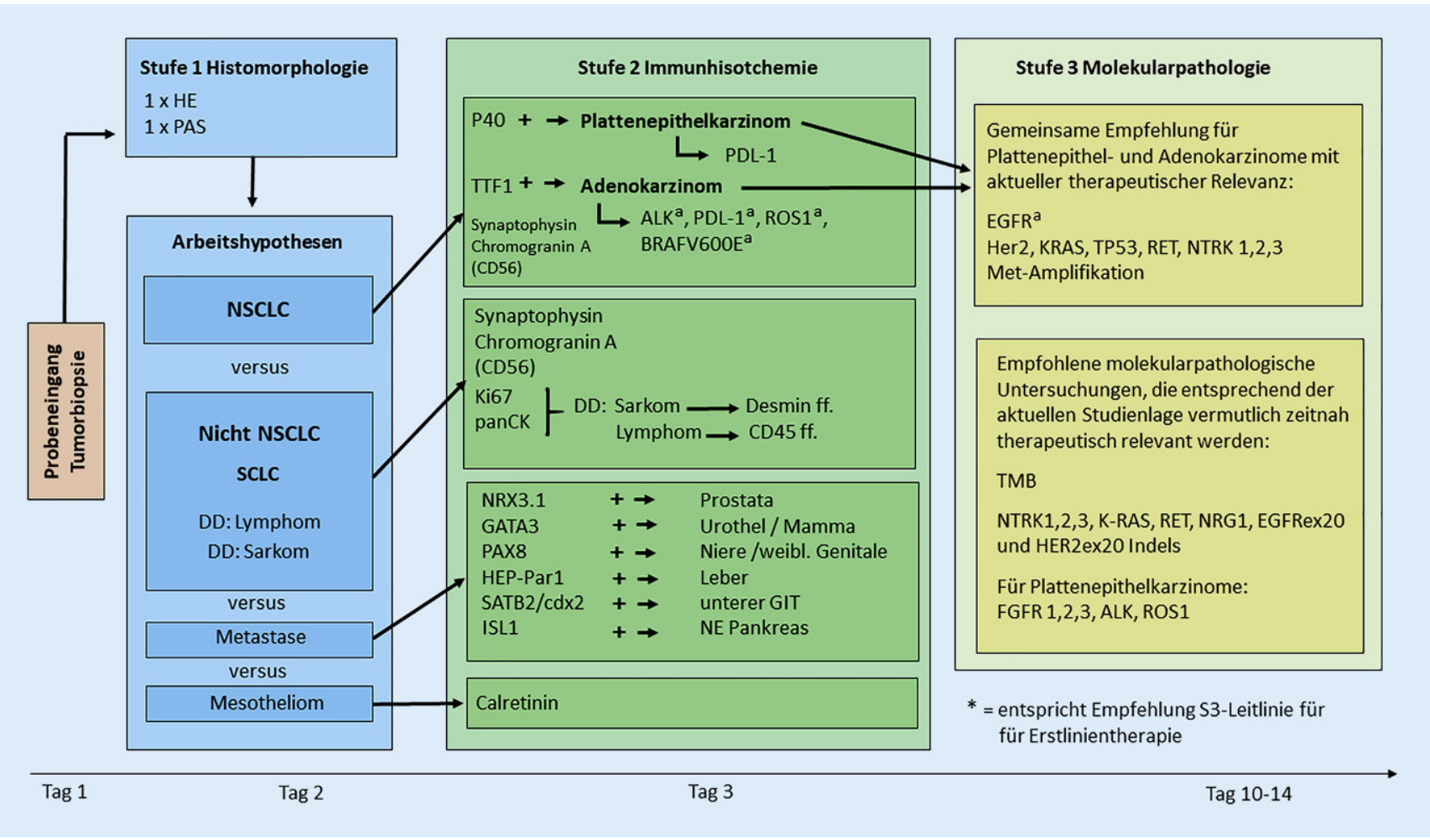

Abb. 1 A 3-Stufen-Schema der pathologischen Diagnostik von Lungenkarzinomen. ${ }^{\mathrm{a}}$ entspricht Empfehlung S3-Leitlinie für Erstlinientherapie. DD Differenzialdiagnose, HE Hämatoxylin-Eosin, NSCLC nichtkleinzelliges Lungenkarzinom, panCK Panzytokeratin, PAS Periodsäure-Schiff, SCLC kleinzelliges Lungenkarzinom, TMB Tumormutationslast

traktion angefertigt; falls genügend Restmaterial verbleibt, ebenfalls im selben Arbeitsgang für die RNA-Extraktion.

Pathologien, die nicht über ein eigenes molekularpathologisches Labor verfügen, sollten die Histologie auf ein Minimum beschränken und lediglich IHC für p40, TTF1, PD-L1 sowie 3 Reserveschnitte für ALK, ROS1 und BRAFV600E bei Adenokarzinomen oder adenosquamösen Karzinomen anfertigen und den Block inklusive eines Schnittpräparates umgehend an ein molekulardiagnostisches Zentrum versenden.

Im 3. Schritt erfolgt die molekulardiagnostische Analytik. Bei Adenokarzinomen müssen mindestens (Minimalanforderung) EGFR, HER2, KRAS, TP53, RET, MET (Ampl.), NTRK1,2,3 bestimmt und optional eine RNA-hybrid-captureSequenzierung auf seltenere Translokationen (MET, NRG1, FGFR1,2,3, EGFRsplice-Varianten) durchgeführt werden. Eine identische Diagnostik erfolgt bei Plattenepithelkarzinomen von Nie- oder Leichtrauchern. Da die klinischen Angaben hierzu in ihrer Qualität stark va- riieren, verläuft die molekulare Analytik konsequenter Weise analog zu Adenokarzinomen. Insbesondere weil es sich bei kleinen Lungenbiopsien, mit Nachweis eines Plattenepithelkarzinoms, stets auch um die Plattenepithelkarzinom-Komponente eines adenosquamösen Karzinoms handeln kann. Diese zeigen in einem deutlich höheren Anteil klinisch relevante EGFR-Mutationen.

Kürzlich wurde der Endpunkt „overall survival“ bei der chemotherapiefreien Erstlinientherapie mit Nivolumab+Ipilimumab für TMBhigh (hohe Tumormutationslast) Tumoren in der CheckMate-227-Studie erreicht [14, 27]. Die Erstlinientherapie mit Nivolumab + Ipilimumab zeigte unabhängig von dem PDL-1-Expressions-Level bei NSCLC-Stadium-IV-Patienten oder NSCLC-Rezidiv ein verlängertes Gesamtüberleben im Vergleich zur Erstlinien-Chemotherapie [15]. Es ist daher $\mathrm{zu}$ erwarten, dass der molekularpathologische Biomarker TMB in naher Zukunft ebenfalls im Schritt 3 zeitnah nach der ersten histologischen Diagnose zu leisten ist. Das würde den Einsatz großer Hybrid-Capture-Panels für die simultane Bestimmung von onkogenen Treibermutationen und TMB in der DNA-Analytik zwingend erfordern.

Der genaue zeitliche Rahmen der histopathologischen Diagnostik sowie die therapeutische Interpretation der Befunde müssen in einem spezialisierten interdisziplinären Tumorboard diskutiert werden. Hier sollte die Reflextestung aller Tumoren auf Marker für die Erstlinienentscheidung in einem zeitnahen Fast-track-Modus vereinbart werden. Nach derzeitiger S3-Leitlinie sind das EGFR, BRAF, ALK, ROS1 und PD-L1 [20]. Parallel dazu sollten innerhalb von 2 Wochen alle weiteren notwendigen Biomarkeranalysen vorliegen.

\section{Immunhistochemische Diagnostik}

Das praktische Vorgehen bei der (sparsamen) Anwendung der IHC-Diagnostik von Lungentumoren wurde vor kurzem von einer IASCL Working Group 
„Best Practices Recommendations for Diagnostic Immunohistochemistry in Lung Cancer" zusammengefasst [34]. Die wichtigsten Empfehlungen hieraus sind:

Falls ein nichtkleinzelliges Karzinom in der Lunge immunhistochemische Marker zur weiteren Subtypisierung erfordert, reichen TTF1 (Klon 8GtG3/1) und p40 aus.

Falls eine eindeutige Keratinisierung, Drüsenbildung oder Muzinbildung in der PAS-Färbung nachweisbar ist, kann auf diese beiden IHC-Untersuchungen sogar ganz verzichtet werden. Keratinfärbungen sollten nicht für die Unterscheidung von Adeno- und Plattenepithelkarzinomen herangezogen werden. Insbesondere CK7 ist nicht spezifisch für Adenokarzinome, sondern wird auch von Plattenepithelkarzinomen exprimiert, sodass CK7 heute zur Subtypisierung von NSCLC nicht mehr empfohlen werden kann. Eine partielle oder fokale TTF1-Reaktion wird als positiv für Adenokarzinome gewertet. Plattenepithelkarzinome sind typischerweise in toto, mindestens aber in $50 \%$ kräftig nukleär positiv für p40. Die p40Färbung ist deutlich spezifischer als p63 und sollte daher immer als plattenepithelialer Marker bevorzugt werden [34].

Nur wenn die Histologie den Verdacht auf eine neuroendokrine Morphologie ergibt, sollten entsprechende zusätzliche Färbungen mit neuroendokrinen Markern durchgeführt werden.

Hierfür wird eine Färbereihe neuroendokriner Marker mit Chromogranin A und Synaptophysin - als wahre Marker neuroendokriner Differenzierung - und ggf. CD56 empfohlen. CD56 sollte stets unter Berücksichtigung der neuroendokrinen Histomorphologie und zusammen mit Ki67 (MIB1) interpretiert werden [34]. Bei SCLC empfiehlt es sich zusätzlich eine Pan-CK-Färbung mit typischem punkt- oder kappenförmigem Färbemuster durchzuführen. Eine Ki67Färbung ist notwendig zur Abgrenzung gegenüber anderen klein-blau-rundzelligen Tumoren (Lymphomen, Sarkomen) und gegenüber niedrig proliferativen NET wie typischen oder atypischen Karzinoiden [31]. Die Diagnose eines SCLC bei einem Nie- oder Leichtraucher sollte

Pathologe 2020 · 41:3-13 https://doi.org/10.1007/s00292-019-00732-4

(c) Der/die Autor(en) 2019

\section{B. J. Wagner $\cdot$ R. Buettner}

\section{Immunhistochemische und molekularpathologische Diagnostik von Lungenkarzinomen}

\section{Zusammenfassung}

Die Therapie der Lungenkarzinome hat binnen der letzten 15 Jahre einen revolutionären Wandel erfahren. Dank zielgerichteter Therapien, insbesondere Thyrosinkinaseinhibitoren (TKI), konnte die Prognose der Patienten deutlich verbessert werden. Aktuell rekrutierende Studien testen unter anderem neue MET-, KRAS-, ROS1-, RET- und NTRK-Inhibitoren. Erste klinische Daten sind vielversprechend, sodass es auch zukünftig Pathologen und Onkologen vor eine Herausforderung stellt, stets alle Facetten differenzierter Therapieentscheidungen im Blick zu halten. Die präzise Klassifikation von Lungenkarzinomen anhand immunhistochemischer und molekularpathologischer Analysen ist heute für die weitere Therapie und Prognose des Patienten von zentraler
Bedeutung. Hinzu kommt, dass neue Technologien der Biopsiegewinnung Minihistologien oder zytologische Proben in höchster diagnostischer Qualität liefern. Aufgrund der Komplexität der notwendigen Diagnostik ist daher ein strategisches Vorgehen erforderlich, um unnötigen Materialverlust zu vermeiden. Ziel dieser Abhandlung ist es, eine aktuelle Übersicht für eine effiziente und zielgerichtete Diagnostik unter Berücksichtigung der aktuellen Studienlage zu erstellen.

Schlüsselwörter

Kleinzelliges Lungenkarzinom · Nichtkleinzelliges Lungenkarzinom - Immunhistochemie . Molekularpathologie $\cdot$ Zielgerichtete Molekulartherapie

\section{Immunohistochemical and molecular pathological diagnosis of lung carcinoma}

\section{Abstract}

The therapy of lung cancer has revolutionarily changed within the last 15 years. The prognosis of patients has dramatically improved due to targeted therapies, for instance tyrosine kinase inhibitors (TKI). Current recruiting studies are testing new MET-, KRAS-, ROS1-, RET- and NTRK-inhibitors. The first clinical data are promising, emphasizing that it remains a future challenge for pathologists and oncologists to keep an eye on all facets of therapy options. Today, precise lung cancer classification via immunohistochemistry and molecular pathology is crucial for the therapy and prognosis of patients. Further, new biopsy technologies lead to very small tumor tissue samples and cytological samples of high diagnostic quality. Therefore, the complexity of diagnosis needs a strategic procedure to minimize loss of tissue material. This assay gives an overview of efficient and target-orientated diagnostic procedures in consideration of current clinical trials.

\section{Keywords}

Small cell lung carcinoma $\cdot$ Non-small cell lung carcinoma $\cdot$ Immunohistochemistry . Molecular pathology · Molecular targeted therapy immer extrem kritisch überprüft werden. Hier sind obligat weitere Marker (z.B. Lymphom, Rhabdomyosarkom, NUT1positives Karzinom) erforderlich.

Bei jedem undifferenzierten Karzinom führen wir eine NUT1-Färbung zum Ausschluss eines NUT1-translozierten Mittellinienkarzinoms durch; ebenso wie bei jedem spindelzelligen Tumor eine Pan-CK- und MET-Färbung zum Ausschluss eines sarkomatoiden NSCLC. Letztlich sollte bei jüngeren Patienten mittels Oct4 und SALL4 ein mediasti- naler bzw. metastasierter Keimzelltumor ausgeschlossen werden.

\section{Welche Untersuchungen eignen sich für die Diagnostik von Tumoren mit Speicheldrüsen- differenzierung?}

Prinzipiell können alle Typen von Speicheldrüsenkarzinomen als primäre Lungenkarzinome oder als Metastasen auftreten. Letzteres kann nur durch eine sorgfältige Anamnese und ein umfas- 


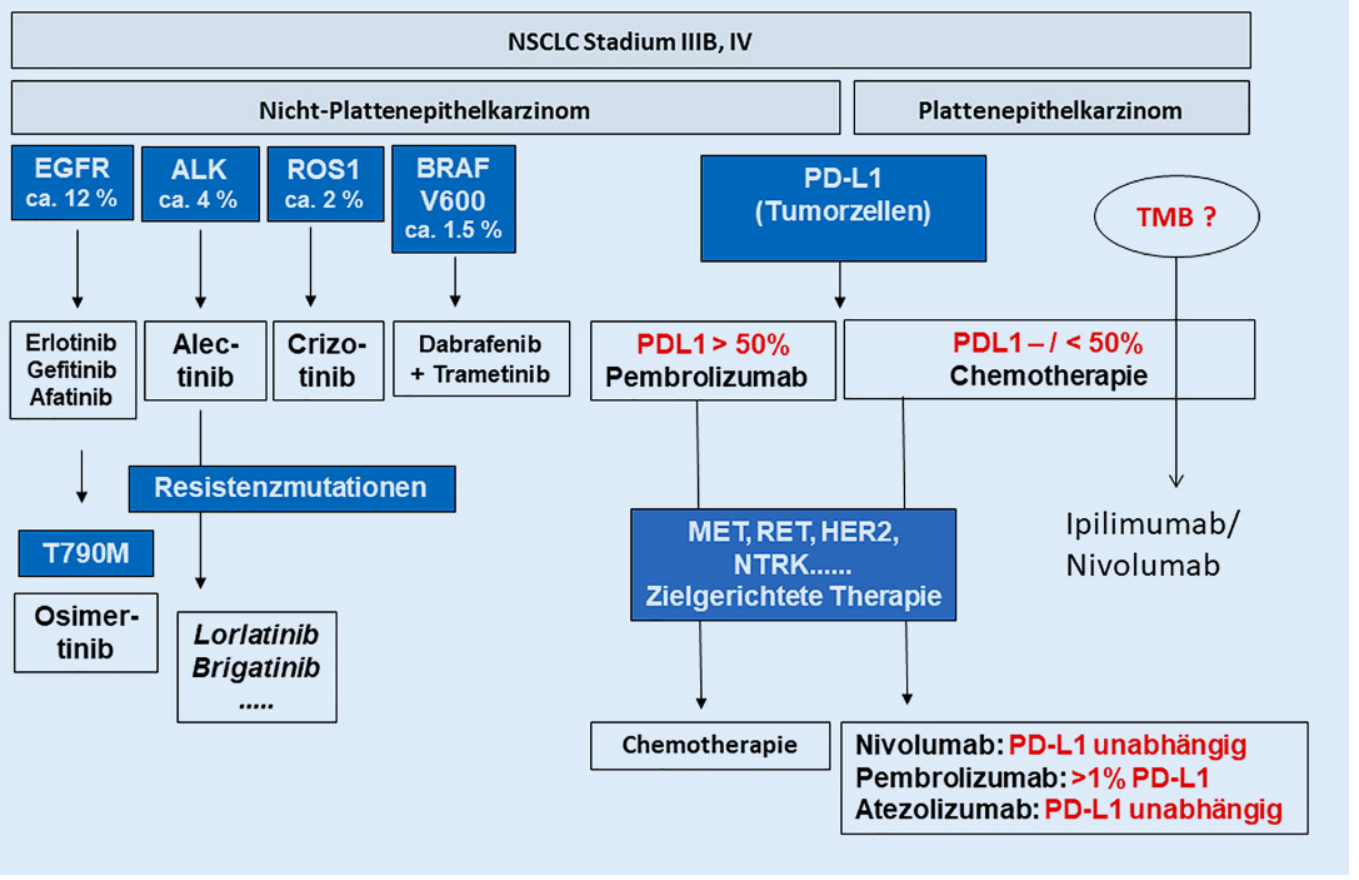

Abb. 2 ム Die systemische Therapie von nichtkleinzelligen Lungenkarzinomen (NSCLC) basiert zunehmend auf der Molekularpathologie. Für die Auswahl des passenden Tyrosinkinaseinhibitors im fortgeschrittenen NSCLC-Stadien ist eine immunhistochemische/molekularpathologische Mutationsanalyse von rezeptorgekoppelten Kinasen und Signaltransduktionskinasen obligat. Die PDL-1-Testung ist bei Adenokarzinomen und Plattenepithelkarzinomen indiziert. Gegebenenfalls wird die Tumormutationslast(TMB)-Bestimmung erforderlich, wenn die CheckMate-227-Studie zeigt, dass nach Erstlinientherapie Nivolumab + Ipilimumab Patienten mit TMBhigh-Tumoren signifikant länger Überleben. NSCLC nichtkleinzelliges Lungenkarzinom, T790M Gatekeepermutation im EGFR-Gen, TMB Tumormutationslast

sendes bildgebendes Staging in einem Tumorboard ausgeschlossen werden. Die mit Abstand häufigsten primär pulmonalen speicheldrüsenanalogen Karzinome sind Mukoepidermoidkarzinome, adenoid-zystische Karzinome und Azinuszellkarzinome. Sehr selten kommen myoepitheliale Karzinome, Klarzellkarzinome und MASC als primäre Lungentumoren vor.

Generell spricht die Expression von TTF1 und/oder Napsin A gegen diese Diagnose und für ein primär pulmonales Adenokarzinom bzw. adenosquamöses Karzinom. Mukoepidermoidkarzinome weisen fast immer eine MAML2-, adenoid-zystische Karzinome eine $M Y B-$, Klarzellkarzinome eine EWS- und MASC eine NTRK3-Translokation auf. Somit sollte bei entsprechender IHC eine FISHDiagnostik oder eine RNA-Sequenzierung zum Nachweis der entsprechenden Translokation eingeleitet werden.
Wie kann man zuverlässig Metastasen von primär pulmonalen malignen Tumoren unterscheiden?

Die Antwort auf diese Frage bleibt bis dato unbefriedigend. Letztlich kann der Pathologe auf sich allein gestellt bei TTF1negativen Adenokarzinomen, NET oder Plattenepithelkarzinomen häufig nicht zwischen primär pulmonalen Karzinomen und Metastasen unterscheiden.

Hilfreich ist ein Panel aus NRX3.1 (Prostata), GATA3 (Urothel, Mamma), Pax8 (Niere, weibliches inneres Genitale), HEP-Par1 (Leber), SATB2/cdx2 (unterer GIT) und ISL1 (NE-Pankreas). Falls TTF1 negativ bleibt, empfehlen wir zunächst eine Rückkopplung mit den klinischen Befunden vorzunehmen, um die IHC gezielt nach dem vermuteten Primärtumor auszubauen. HPV-Positivität spricht sehr stark für einen Primärtumor im oberen Aerodigestivtrakt oder Genital-/Analbereich.

\section{Zytologische Tumordiagnostik}

Prinzipiell ist die gesamte morphologische und molekulare Diagnostik sowie IHC-Diagnostik von Lungentumoren auch an zytologischen (Ausstrich-)Präparaten möglich. Ausgenommen hiervon sind IHC-Membranfärbungen, wie z.B. PD-L1, die besser an Zytoblockpräparaten gelingen $[9,13]$. Da bei Ausstrichund Zytospinpräparaten keine Schnittund Formalinartefakte auftreten, ist die $\mathrm{zu}$ Sequenzierungen gewonnene DNA und RNA häufig von besserer Qualität. Wir empfehlen jedoch, wenn möglich, auch ein Zytoblockpräparat anzufertigen, da dann Techniken wie FISHAnalysen nicht nochmals standardisiert werden müssen. Generell versuchen wir stets, bei Ergüssen oder Aspirationsmaterialien das gesamte Zellmaterial als Zytoblock einzubetten. 


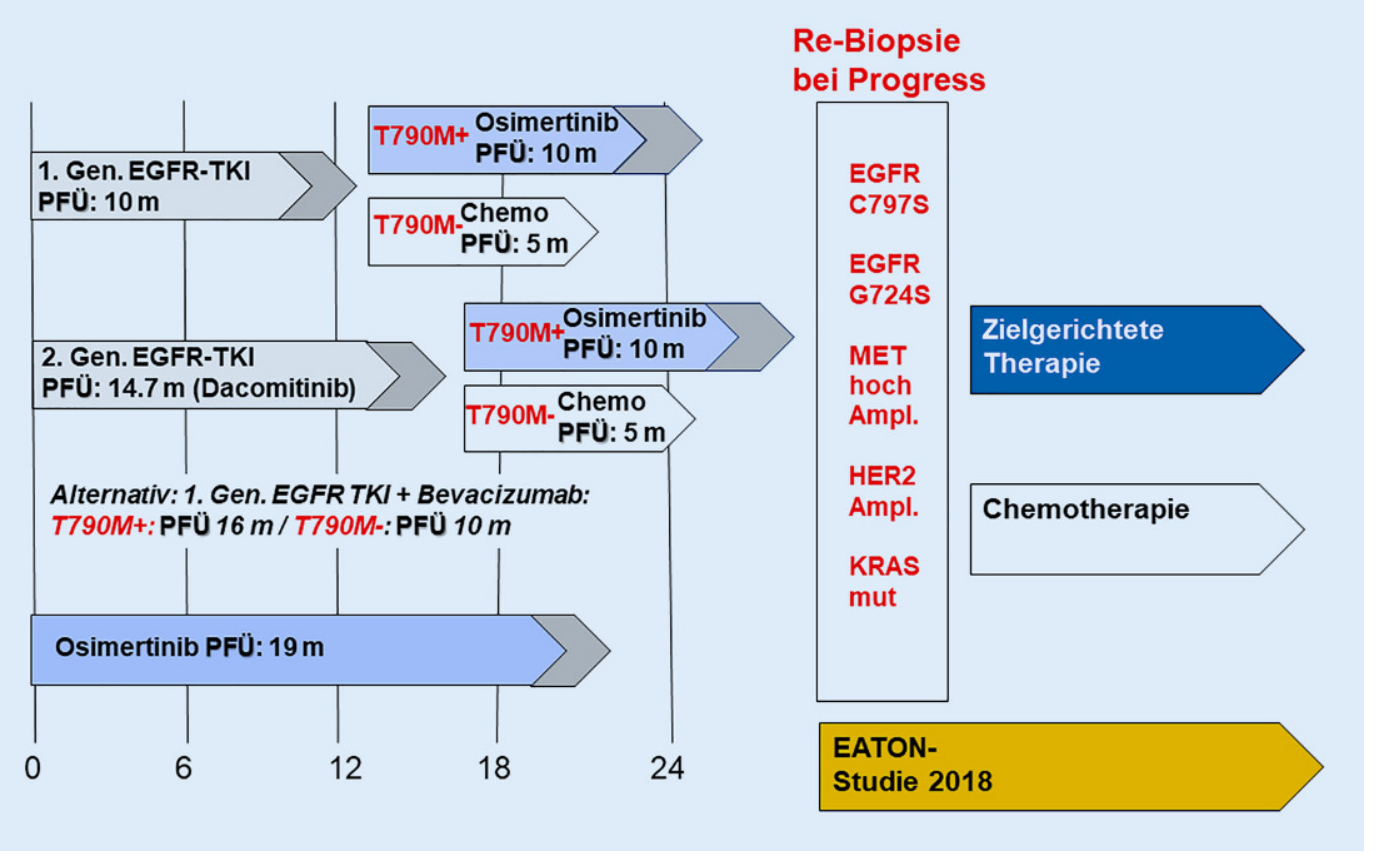

Abb. 3 ॥ Zweitlinientherapien nach Osimertinib bei EGFR-mutierten nichtkleinzelligen Lungenkarzinomen (NSCLC). (1.) Es muss die Fortsetzung von Osimertinib, die Kombination mit anderen Tyrosinkinaseinhibitoren (TKIs) oder der Wechsel zur Chemotherapie von dem Vorliegen der T790M-Mutation im EGFR-Gen abhängig gemacht werden. (2.) Es sollte bei Tumorprogress eine Rebiopsie erfolgen, um weitere zielgerichtete Therapiemöglichkeiten als Alternative zur Chemotherapie zu erfassen. Die Eaton-Studie der Lung Cancer Group Cologne (LCGC) ist eine multizentrische, offene Phase-I-Dosiseskalationsstudie mit EGF816 und Trametinib (Kontakt: https://lungcancergroup.de/studie/eaton/). PFÜ Progressionsfreies Überleben, $m$ Monate, T790M Gatekeepermutation im EGFR-Gen

\section{Liquiddiagnostik anhand zirkulierenden Tumor-DNA im peripheren Blut}

Obwohl es einzelne Berichte gibt, dass sich aus der Kombination suggestiver, bildgebender Befunde und der Sequenzierung von ctDNA im Blut Lungentumoren erfolgreich typisieren und mit zielgerichteten Therapien behandeln lassen $[2,19]$, birgt diese Praxis auch die Gefahr einer Fehlinterpretation für den Patienten. Erstens ist eine präzise, histopathologische Tumordiagnostik nur am Gewebeschnitt bzw. zytologischen Präparat möglich (Plattenepithelkarzinom vs. Adenokarzinom etc.). Dies ist für die Auswahl der Chemotherapie auch bei kombinierter Immunchemotherapie essenziell, da eine Kombination mit Pemetrexed bei Adenokarzinomen überlegen ist. Zweitens gibt es inzwischen Berichte, dass die Detektion einer Mutation (z.B. von KRAS oder TP53) aufgrund klonaler, hämatopoetischer Aberrationen zu falsch positiven Ergebnissen führen kann [18]. Daher fordern wir in der Primärdiagnos- tik eine umfassende histologische und molekularpathologische Tumordiagnostik sowie IHC-Tumordiagnostik. Diese sollte nur in seltenen Ausnahmesituationen (wiederholt fehlgeschlagene Histologie, Gefährdung des Patienten bei Blutungsneigung etc.) durch andere letztlich weniger aussagekräftige Behelfsmethoden ersetzt werden.

Die Liquiddiagnostik von ctDNA hat ihre große Domäne im Monitoring von Patienten mit bekannten Tumormutationen unter Therapie. Sowohl das Ansprechen auf Therapien wie auch die Entwicklung von Resistenzen können durch die Liquiddiagnostik unterstützt werden [17]. Obwohl derzeit aus kommerziellen Gründen eine TMB-Diagnostik am peripheren Blut propagiert wird [22], ist die Bestimmung auch dieses komplexen $\mathrm{Pa}$ rameters mit zahlreichen Testverfahren zusammen mit den onkogenen Treibermutationen praktikabler und auch ökonomischer durchzuführen.

\section{Molekulare Diagnostik}

Eine (nicht ganz vollständige) Zusammenfassung der Erstlinien-Therapieoptionen bei systemisch fortgeschrittenen Lungenkarzinomen findet sich in (• Abb. 2). Bei der gegenwärtigen dynamischen Entwicklung von Lungenkrebstherapien werden bei Erscheinen dieses Artikels möglicherweise eine Reihe weitere, effektive Erstlinientherapien zugelassen sein (NTRK-, RET-, METTKI oder auch chemotherapiefreie Kombinationstherapien mit zwei dualen Immuncheckpoint-Inhibitoren). Bezüglich der bislang obligat erforderlichen Biomarker für Erstlinientherapien (EGFR, BRAF, ALK, ROS1, PD-L1) verweisen wir auf unsere Zusammenfassung der Kommentierung der gegenwärtigen S3Leitlinie zu Lungenkrebs [20]. Aufgrund des begrenzten Umfangs des vorliegenden Artikels kann hier nur ein Brevier mit Zwischenstand September 2019 gegeben werden. 


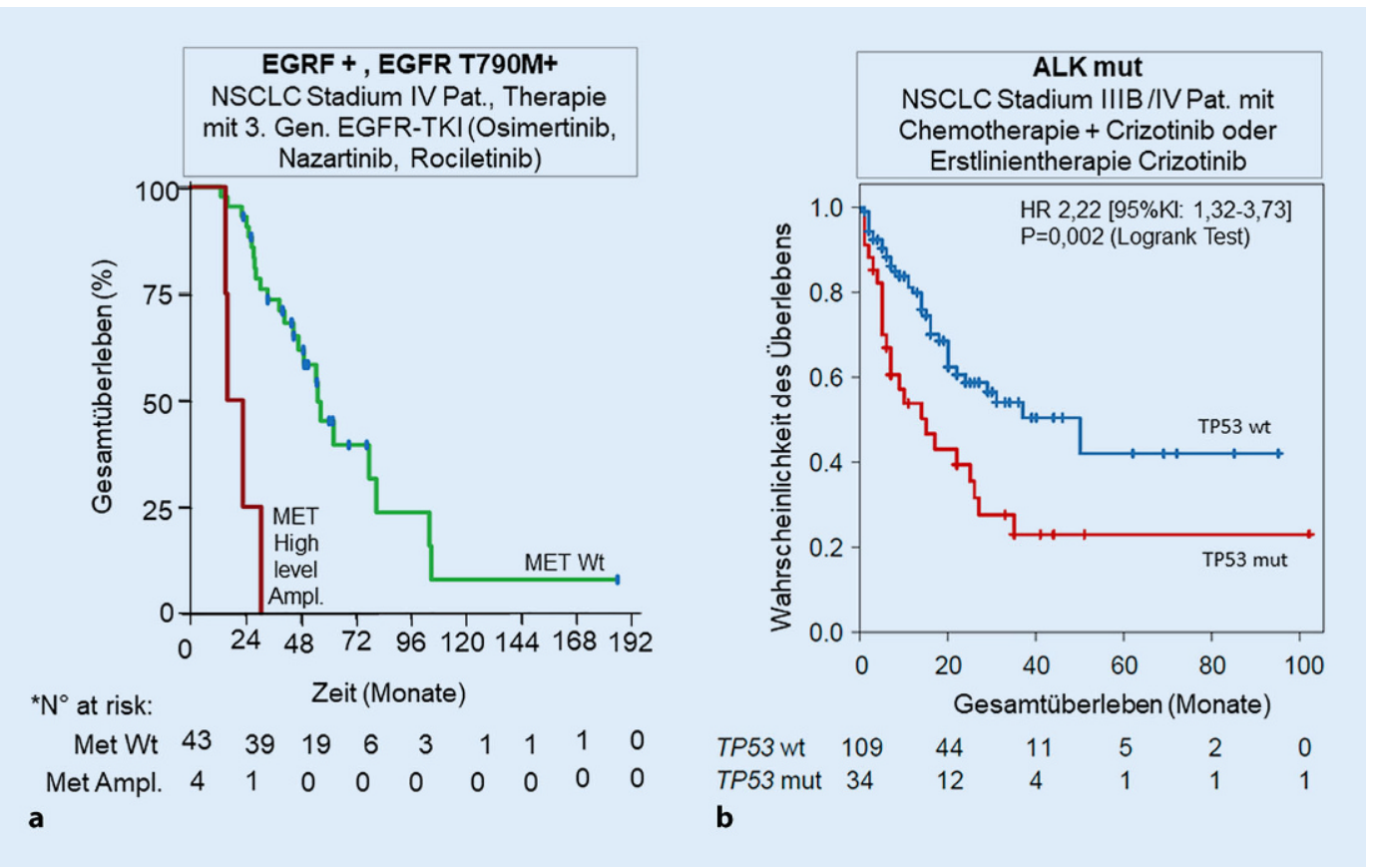

Abb. 4 A Einfluss von MET- und P53-Mutationen auf das Gesamtüberleben von EGFR- und ALK- mutierten Patienten mit nichtkleinzelligem Lungenkarzinom (NSCLC) in Stadium IIIB/IV. (a) EGFR-T790M-mutierte NSCLC-Patienten, die im Stadium IV mit eine Resistenz gegen EGFR-Inhibitoren der 1. und 2. Generation zeigen, haben unter Therapie mit einem EGFR Inhibitor der 3. Generation bei gleichzeitiger MET-high-level-Amplifikation eine deutlich geringere Überlebensrate im Vergleich zu MET-Wildtyp. (@ Michels et al. [unveröffentlicht]) (b) ALK-mutierte Patienten in NSCLC-Stadium IIIB/IV mit Erstlinientherapie Chemotherapie + Crizotinib oder Crizotinib mono haben bei gleichzeitiger TP53-Mutation eine deutlich geringere Überlebensrate im Vergleich zu TP53-Wildtyp. (Eigene Daten in Anlehnung an Kron et al. [21], jeweils nNGM). ${ }^{N}$ at risk Patientenanzahl, die zu dem Zeitpunkt noch nicht tot/nicht zensiert sind, Ampl. Amplifikation, NSCLC nichtkleinzelliges Lungenkarzinom, T790M Gatekeepermutation im EGFR-Gen, HR Hazard Ratio, KI Konfidenzintervall, mut mutiert, TKI Tyrosinkinaseinhibitor, wt Wildtyp

\section{EGFR-mutierte Adenokarzinome}

Die Standardtherapie bei aktivierenden EGFR-Mutationen in Exon 19 und 21 ist seit der FLAURA-Studie der Drittlinieninhibitor Osimertinib [30]. Alternativ kann der Einschluss in eine klinische Studie mit neueren Drittlinieninhibitoren (z.B. EGFR816) im Rahmen des nationalen Netzwerkes Genomische Medizin (nNGM, https://www.nngm.de) erfolgen. Die klinischen Daten zeigen, dass dieses Vorgehen eindeutig der sequenziellen Therapie mit einem Erst- bzw. Zweitlinieninhibitor (Gefitinib, Erlotinib oder Afatinib) und anschließender Zweittherapie je nach Resistenzmechanismus überlegen ist [29]. Hinzu kommt, dass viele Patienten nach Versagen der Erstlinientherapien nicht erneut getestet werden und somit die Chance der resistenzadaptierten Zweitlinientherapie verloren geht. Heute sind zahlreiche gut therapierbare Resistenzmechanismen nach Versagen der Osimertinib-Thera- pie bekannt [10]. In jedem Fall sollte bei Versagen eine Rebiopsie bzw. Liquiddiagnostik durchgeführt werden, um zu prüfen, welche Zweitlinientherapie nach Osimertinib durchgeführt werden kann (- Abb. 3).

Ausnahmen von diesem Vorgehen sind Indelmutationen in Exon 20 von EGFR (und Exon 20 von HER2), die auf herkömmliche EGFR-Inhibitoren oft nur schlecht oder gar nicht ansprechen. Hier besteht im nNGM die Möglichkeit eines Studieneinschlusses mit dem speziell für diese Mutationen entwickelten Inhibitor TAK788 (ASCO annual meeting 2019; Abstract 9007, https://abstracts.asco. org; Kontakt über nNGM, https://www. nngm.de). Auch für die seltenen EGFRExon-18- und aktivierenden Exon-20Punktmutationen bitten wir um einen Einschluss in eine Beobachtungsstudie des nNGM, um verlässlichere klinische Daten zu erheben, für welche Mutationen welcher Inhibitor am geeignetsten ist.

\section{BRAF-V600E-Mutationen}

Hierfür sind mehrere Kombinationstherapien aus BRAF- und MEK-Inhibitoren für die Erstlinie zugelassen. Die Resistenzmechanismen bei BRAF-mutierten Karzinomen sind komplex und erlauben keine Zweitlinientherapie mit einem TKI. Daher sollte obligat der PD-L1-Status direkt mitbestimmt werden, um eine adaptierte Immun- oder Immunchemotherapie nach Versagen der Erstlinientherapie einzuleiten.

\section{ALK-Translokationen}

Auch hier hat es einen Wandel des Erstlinien-TKI gegeben, insofern klinische Studien eindeutig belegen, dass der Inhibitor Alectinib der bisherigen Standardtherapie von Crizotinib überlegen ist. Die Daten des Alex-Trials zeigen, dass insbesondere der Progress von ZNS-Metastasen unter Alectinib deutlich geringer ist, weil dieser Inhibitor im Gegensatz zu 


\begin{tabular}{|c|c|c|c|c|c|c|}
\hline & Academic Panel & Illumina & Thermo & NEOplus v2 & Qiagen & F one \\
\hline Exonregion & $1,1 \mathrm{Mb}$ & $1,9 \mathrm{Mb}$ & $1,7 \mathrm{Mb}$ & $1,2 \mathrm{Mb}$ & $1,3 \mathrm{Mb}$ & $1,1 \mathrm{Mb}$ \\
\hline Drivermutationen & $\checkmark$ & $\checkmark$ & Nein & $\checkmark$ & Keine Fusionen & $\checkmark$ \\
\hline Mikrosatelliten & $\checkmark$ & $\checkmark$ & Nein & $\checkmark$ & $\checkmark$ & $\checkmark$ \\
\hline Input (Probenmenge) & $50 \mathrm{ng}$ & $40 \mathrm{ng}$ & $20 \mathrm{ng}$ & $50 \mathrm{ng}$ & $10-40 \mathrm{ng}$ & $200 \mathrm{ng}$ \\
\hline Negative Prädikationen & $\checkmark$ & $\checkmark$ & Unbekannt & $\checkmark$ & $\checkmark$ & $\checkmark$ \\
\hline Resistenzmutationen & $\checkmark$ & $\checkmark$ & Unbekannt & $\checkmark$ & $\checkmark$ & $\checkmark$ \\
\hline Repair-Pathway-Gene & $\checkmark$ & $\checkmark$ & Unbekannt & $\checkmark$ & $\checkmark$ & $\checkmark$ \\
\hline Sensitivität & Unbekannt & Unbekannt & Unbekannt & Unbekannt & Unbekannt & $78,6 \%$ \\
\hline Spezifität & Unbekannt & Unbekannt & Unbekannt & Unbekannt & Unbekannt & $70,6 \%$ \\
\hline Wet-lab-getestet & Aktuell andauernd & Aktuell andauernd & $\checkmark$ & $\checkmark$ & Aktuell andauernd & $\checkmark$ \\
\hline Testkit lokal verfügbar & $\checkmark$ & $\checkmark$ & $\checkmark$ & $\checkmark$ & $\checkmark$ & Nein \\
\hline Bioinformatik lokal verfügbar & Nein & Bisher nicht getestet & $\checkmark$ & Nein & Bisher nicht getestet & Nein \\
\hline
\end{tabular}

Crizotinib nicht effektiv aus dem Liquor heraustransportiert wird [25]. Zudem ist die Rate an therapiefähigen Resistenzmutationen nach Alectinib mit über $50 \%$ der Fälle deutlich höher, sodass wir immer eine Rebiopsie/Liquiddiagnostik empfehlen, um den effektivsten TKI für eine Zweitlinientherapie auszuwählen [12].

Daten des nNGM zeigen, dass sowohl Patienten mit EGFR-Mutationen wie auch mit $A L K$-Translokationen eine $50 \%$ ige Überlebensrate von 5 Jahren und mehr erreichen, falls 2 Therapielinien mit TKI durchgeführt werden, sodass dieses diagnostische Vorgehen von großer klinischer Relevanz ist [21]. Neuere klinische Daten des nNGM belegen allerdings, dass $A L K$-translozierte Karzinome mit synchroner TP53-Mutation deutlich schneller in einen TKI-resistenten Progress mit ungünstiger chromosomaler Instabilität kommen [1]. Wir empfehlen daher auch bei Nachweis einer Translokation, die umfassende genomische Charakterisierung eines jeden $\mathrm{Tu}$ mors sowohl in der Primärsituation wie auch bei Resistenzbestimmung anzustreben (• Abb. 4).

\section{ROS-Translokation}

Auch für diese Gruppe von etwa 1,6 \% der Lungenadenokarzinome stehen mehrere effektive Erst- und Zweitlinien TKIs zur Verfügung (Crizotinib, Entrectinib, Lorlatinib etc.) [3]. Wir empfehlen ein primäres Screening von NSCLC mittels IHC [8] und bei Progress nach primärer TKITherapie in jedem Fall eine Rebiopsie/
Liquiddiagnostik zur Ermittlung einer möglichen Zweitlinientherapie. Es gibt wahrscheinlich kaum andere Tumortypen als ROS1-translozierte Adenokarzinome, die so stark von einer zielgerichteten Therapie mit TKI profitieren, wie diese zahlenmäßig beträchtliche Patientengruppe [24].

\section{Neue Tyrosinkinaseinhibitoren für seltene Fusionen}

Bei Erscheinen dieses Manuskriptes liegen möglicherweise bereits Zulassungen für mehrere TKI in der Erstlinientherapie bei NSCLC mit Fusionen in den Rezeptoren RET (ca. 0,5-0,8\% der Adenokarzinome), NTRK-1,-2 und -3 (0,1-0,2\% der Adenokarzinome) vor. Weitere klinische Studien und Off-label-Beobachtungen im nNGM gibt es für Tumoren mit Fusionen in FGFR-1,-2 und -3 (ca. 1\% der NSCLC) und dem Rezeptorliganden NRG1. Resistenzmechanismen sind hierbei kaum bekannt, sodass wir bitten, die Rebiopsien nach Therapie im Rahmen von nNGM Studien durchführen zu lassen.

\section{MET-Alterationen}

Für den Membranrezeptor MET (Ligand: "hepatocyte growth factor") sind 4 wichtige genetische Aberrationen bekannt: 1) Deletionen in Intronbereichen um Exon 14, die zu Spleissvarianten ohne Exon 14 und zu längerem Verweilen von MET in der Zellmembran führen („exon14 skipping mutations"),
2) Amplifikationen, 3) Genfusionen und 4) kinaseaktivierende Punktmutationen [11, 26, 28]. In der Zulassungsstudie mit dem MET-Inhibitor Capmatinib werden derzeit Genamplifikationen und Exon14-skipping-Mutationen eingeschlossen (Geometry Trial der nNGM). Daneben besteht für alle anderen Patienten die Möglichkeit einer Off-label-Therapie mit dem MET-Inhibitor Crizotinib im Rahmen von nNGM.

\section{KRAS-aktivierende Mutationen}

Die bislang größte Patientengruppe mit onkogenen Treibermutationen in Lungenkarzinomen in Europa sind solche mit KRAS-Mutationen. Mehrere Inhibitoren mit irreversibler Bindung an die Aminosäure Cystein sind in klinischer Prüfung. Diese Inhibitoren wirken prinzipiell nur, wenn die KRAS-Mutation $\mathrm{zu}$ einem Cysteinaustausch führt, wie z.B. bei der in Lungenkarzinomen sehr häufigen KRAS-G12C-Mutation. Die am weitesten fortgeschrittene Studie der Firma AMGEN mit dem Inhibitor AMG wird auch in Kürze in Deutschland Patienten rekrutieren und hat in vorläufigen Daten mit einer Ansprechrate von über $50 \%$ bei multipel vorbehandelten Patienten große Erwartungen geweckt (ASCO annual meeting 2019; Abstract 3003, https://abstracts.asco.org). Daher ist die genaue Mutationsbestimmung in RAS-Genen von hoher Bedeutung für die Patienten. Es deutet sich hier ein neuer bahnbrechender Fortschritt in der 


\begin{tabular}{|c|c|}
\hline \multicolumn{2}{|c|}{ Abkürzungen } \\
\hline Ampl. & Amplifiziert \\
\hline BRAF & $\begin{array}{l}\text { Serin/Threonin-Proteinkinase } \\
\text { B-Raf }\end{array}$ \\
\hline$C K$ & Zytokeratin \\
\hline$C T$ & Computertomographie \\
\hline ctDNA & Zirkulierende Tumor-DNA \\
\hline$E B B$ & Endobronchiale Biopsie \\
\hline EBUS-TBNA & $\begin{array}{l}\text { Endobronchiale, ultraschall- } \\
\text { gesteuerte transbronchiale } \\
\text { Nadelaspiration }\end{array}$ \\
\hline EGFR & $\begin{array}{l}\text { „Epidermal growth factor } \\
\text { receptor" }\end{array}$ \\
\hline$E v G$ & Elastica van Gieson \\
\hline FGFR1-3 & $\begin{array}{l}\text { „Fibroblast growth factor } \\
\text { receptor } 1-3 \text { " }\end{array}$ \\
\hline FISH & $\begin{array}{l}\text { Fluoreszenz-in-situ-Hybridi- } \\
\text { sierung }\end{array}$ \\
\hline$H E$ & Hämatotoxylin-Eosin \\
\hline HER2 & $\begin{array}{l}\text { Humaner-epidermaler-Wachs- } \\
\text { tumsfaktor-Rezeptor } 2\end{array}$ \\
\hline$H P V$ & Humane Papillomviren \\
\hline IASLC & $\begin{array}{l}\text { International Association for the } \\
\text { Study of Lung Cancer }\end{array}$ \\
\hline$I H C$ & $\begin{array}{l}\text { Immunhistochemie, immunhis- } \\
\text { tochemisch }\end{array}$ \\
\hline Indel & $\begin{array}{l}\text { Verschmelzung aus Insertion } \\
\text { und Deletion }\end{array}$ \\
\hline KI & Konfidenzintervall \\
\hline
\end{tabular}

Therapie dieser bisher sehr ungünstigen Mutation an.

\section{Mutationen in pulmonalen Plattenepithelkarzinomen}

Bislang werden nach unseren Beobachtungen Patienten mit pulmonalen Plattenepithelkarzinomen in Deutschland kaum molekular getestet (mit Ausnahme der Bestimmung von PD-L1). Die in diesen Tumoren häufigen Mutationen in TP53, PIK3CA, PTEN, NFE2L2 u.a. eignen sich zwar zur molekularen Subtypisierung [7], aber entsprechende klinische Studien sind bislang fehlgeschlagen. Nach unseren Erfahrungen weisen aber fast $2 \%$ dieser Patienten therapeutisch hochrelevante genetische Aberrationen (Fusionen, Mutationen

\begin{tabular}{|c|c|}
\hline \multicolumn{2}{|c|}{ Abkürzungen (Fortsetzung) } \\
\hline LCNEC & $\begin{array}{l}\text { Großzelliges neuroendokrines } \\
\text { Karzinom }\end{array}$ \\
\hline MASC & $\begin{array}{l}\text { Mammaanaloges sekretorisches } \\
\text { Karzinom }\end{array}$ \\
\hline mut & Mutiert \\
\hline NEK & Neuroendokrine Karzinome \\
\hline NET & Neuroendokrine Tumoren \\
\hline$n N G M$ & $\begin{array}{l}\text { Nationales Netzwerk Genomi- } \\
\text { sche Medizin }\end{array}$ \\
\hline NSCLC & $\begin{array}{l}\text { Nichtkleinzelliges Lungenkarzi- } \\
\text { nom }\end{array}$ \\
\hline PAS & Periodsäure-Schiff \\
\hline Pat. & Patient \\
\hline$P D-L 1$ & „Programmed death-ligand 1“ \\
\hline QulP & Qualitätsinitiative Pathologie \\
\hline$S C L C$ & Kleinzelliges Lungenkarzinom \\
\hline T790M & $\begin{array}{l}\text { Gatekeepermutation im } \\
\text { EGFR-Gen }\end{array}$ \\
\hline$T B B$ & Transbronchiale Biopsie \\
\hline TKI & Thyrosinkinaseinhibitoren \\
\hline$T M B$ & Tumormutationslast \\
\hline TTF1 & $\begin{array}{l}\text { "Transcription termination } \\
\text { factor } 1 "\end{array}$ \\
\hline WHO & Weltgesundheitsorganisation \\
\hline$W t$ & Wildtyp \\
\hline ZNS & Zentrales Nervensystem \\
\hline
\end{tabular}

und Amplifikationen in EGFR, $A L K$, ROS, FGFR-1,-2,-3, MET, KRAS) auf, sodass wir empfehlen, alle Patienten mit Plattenepithelkarzinomen darauf $\mathrm{zu}$ screenen. Die Rate an therapeutisch relevanten Mutationen in adenosquamösen Karzinomen ist im Gegensatz zu rein squamösen Karzinomen sehr hoch [33], sodass dieser Tumortyp immer umfassend getestet werden sollte.

\section{Tumormutationslast}

In 2 klinischen Studien (CM26, Mystic) wurde eine Rationale erarbeitet, die darauf hindeutet, dass Tumoren mit einer hohen Zahl an Missensemutationen vom Immunsystem besser erkannt werden und somit von einer chemotherapiefreien kombinatorischen Therapie mit 2 Immuncheckpoint-Inhibitoren am meisten profitieren [6]. Daraufhin wurde die prospektive Studie CM227 gestartet, deren Daten zum Gesamtüberleben im Herbst 2019 erwartet werden. Sollten diese die vorläufigen Daten zum progressionsfreien Überleben bestätigen, ist mit einer zeitnahen Zulassung der Therapie (Ipilimumab/Nivolumab) in der Erstlinie zu rechnen, sodass eine rasche Analyse der Mutationslast in hierfür spezialisierten molekularpathologischen Labors erforderlich ist. Daten aus dem nNGM und der QuIP zeigen, dass hierfür eine Reihe unterschiedlicher Testverfahren geeignet sind und zuverlässige Ergebnisse liefern können [4], sodass ein Versand an Labors außerhalb der Pathologie zur Bestimmung von TMB nicht sinnvoll ist. Allerdings ist hierfür eine komplexe Analysestruktur mit Bereitstellung einer leistungsfähigen Bioinformatik und einer klinischen Integration der $\mathrm{Be}$ fundinterpretation notwendig, wie sie derzeit nur in einigen nNGM-Zentren zur Verfügung steht (• Tab. 1).

\section{Fazit für die Praxis}

- Neue Biopsietechniken führen zu Tumorproben von hoher Qualität mit trotz abnehmender Gewebemenge, sodass eine materialschonende und gleichzeitig zielgerichtete pathologische Diagnostik erforderlich ist.

- Zur effizienten und materialschonenden Diagnostik empfehlen wir eine 3-Stufen-Diagnostik.

- Obligat erforderliche Biomarker für die Erstlinientherapien aller nichtkleinzelligen Lungenkarzinome entsprechend der aktuellen S3-Leitlinie sind: EGFR, BRAF, ALK, ROS1, PDL1. Wir empfehlen dabei zusätzlich auch alle Plattenepithelkarzinome zu testen.

- Weitere molekularpathologische Untersuchungen, die bezüglich der aktuellen Studienlage vermutlich zeitnah eine therapeutische Relevanz erfahren und wir somit zu testen empfehlen:

- NTRK1,2,3, KRAS, RET, NRG1, EGFRex20 und HER2ex20-Indels,

- Tumormutationslast, 
Hier steht eine Anzeige.

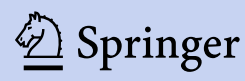




\section{- für Plattenepithelkarzinome: FGFR 1,2,3, ALK, ROS1.}

\section{Die Therapie des Lungenkarzinoms unterliegt weiterhin einem rasanten Wandel und stellt für den Pathologen und Onkologen eine Herausfor- derung dar, stets "up to date" zu bleiben.}

\section{Korrespondenzadresse

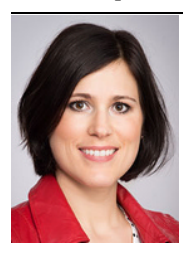 \\ Dr. med. \\ Britta Janina Wagner \\ Institut für Pathologie, Universitätsklinikum und Centrum für Integrierte Onkologie (ClO) Köln Kerpener Straße 62, 50937 Köln, Deutschland britta.wagner@uk-koeln.de}

\section{Einhaltung ethischer Richtlinien}

Interessenkonflikt. B. J. Wagner und R. Buettner geben an, dass kein Interessenkonflikt besteht.

Für diesen Beitrag wurden von den Autoren keine Studien an Menschen oder Tieren durchgeführt. Für die aufgeführten Studien gelten die jeweils dort angegebenen ethischen Richtlinien.

Open Access. Dieser Artikel wird unter der Creative Commons Namensnennung 4.0 International Lizenz veröffentlicht, welche die Nutzung, Vervielfältigung Bearbeitung, Verbreitung und Wiedergabe in jeglichem Medium und Format erlaubt, sofern Sie den/die ursprünglichen Autor(en) und die Quelle ordnungsgemäß nennen, einen Link zur Creative Commons Lizenz beifügen und angeben, ob Änderungen vorgenommen wurden.

Die in diesem Artikel enthaltenen Bilder und sonstiges Drittmaterial unterliegen ebenfalls der genannten Creative Commons Lizenz, sofern sich aus der Abbildungslegende nichts anderes ergibt. Sofern das betreffende Material nicht unter der genannten Creative Commons Lizenz steht und die betreffende Handlung nicht nach gesetzlichen Vorschriften erlaubt ist, ist für die oben aufgeführten Weiterverwendungen des Materials die Einwilligung des jeweiligen Rechteinhabers einzuholen.

Weitere Details zur Lizenz entnehmen Sie bitte der Lizenzinformation auf http://creativecommons.org/ licenses/by/4.0/deed.de.

\section{Literatur}

1. Alidousty C, Baar T, Martelotto LG, Heydt C, Wagener S, Fassunke J, Duerbaum N, Scheel AH, Frank S, Holz B, Binot E, Kron A, MerkelbachBruse S, Ihle MA, Wolf J, Buettner R, Schultheis AM (2018) Genetic instability and recurrent MYC amplification in ALK-translocated NSCLC: a central role of TP53 mutations. JPathol 246:67-76

2. Bouhlel L, Hofman V, Maschi C, Ilie M, Allegra M, Marquette $\mathrm{CH}$, Audigier-Valette $\mathrm{C}$, Thariat J, Hofman P (2017) The liquid biopsy: a tool for a combined diagnostic and theranostic approach for care of a patient with late-stage lung carcinoma presenting with bilateral ocular metastases. Expert Rev Anticancer Ther 17:1087-1092

3. Bubendorf $L$, Buttner R, Al-Dayel F, Dietel M Elmberger G, Kerr K, Lopez-Rios F, Marchetti A, Oz B, Pauwels P, Penault-Llorca F, Rossi G, Ryska A Thunnissen E (2016) Testing for ROS1 in non-small cell lung cancer: a review with recommendations. Virchows Arch 469:489-503

4. Buttner R, Longshore JW, Lopez-Rios F, Merkelbach-Bruse S, Normanno N, Rouleau E, PenaultLlorca F (2019) Implementing TMB measurement in clinical practice: considerations on assay requirements. ESMO Open 4:e442

5. Cancer Genome Atlas Research Network (2014) Comprehensive molecular profiling of lung adenocarcinoma. Nature 511:543-550

6. Carbone DP, Reck M, Paz-Ares L, Creelan B, Horn L, Steins M, Felip E, van den Heuvel MM, Ciuleanu TE, Badin F, Ready N, Hiltermann TJN, Nair S, Juergens R, Peters S, Minenza E, Wrangle JM Rodriguez-Abreu D, Borghaei $\mathrm{H}$, Blumenschein GR Jr., Villaruz LC, Havel L, Krejci J, Corral JJ, Chang H, Geese WJ, Bhagavatheeswaran P, Chen AC, Socinski MA, CheckMate 026 Investigators (2017) First-line nivolumab in stage IV or recurrent nonsmall-cell lung cancer. NEngl JMed 376:2415-2426

7. Clinical Lung Cancer Genome Project, Network Genomic Medicine (2013) A genomics-based classification of human lung tumors. Sci Transl Med 5:209ra153

8. Conde E, Hernandez S, Martinez R, Angulo B, De Castro J, Collazo-Lorduy A, Jimenez B, Muriel A, Mate JL, Moran T, Aranda I, Massuti B, Rojo F, Domine M, Sansano I, Garcia F, FelipE, Mancheno N, Juan O, Sanz J, Gonzalez-Larriba JL, AtienzaCuevas L, Arriola-Arellano E, Abdulkader I, GarciaGonzalez J, Camacho C, Rodriguez-Abreu D, Teixido C, Reguart N, Gonzalez-Pineiro A, LazaroQuintela M, Lozano MD, Gurpide A, GomezRoman J, Lopez-Brea M, Pijuan L, Salido M, Arriola E, Company A, Insa A, Esteban-Rodriguez I, Saiz M, Azkona E, Alvarez R, Artal A, Plaza ML, Aguiar D, Enguita $A B$, Benito $A$, Paz-Ares $L$, Garrido $P$, Lopez-Rios F (2019) Assessment of a new ROS1 immunohistochemistry clone (SP384) for the identification of ROS1 rearrangements in nonsmall cell lung carcinoma patients: the ROSING study. J Thorac Oncol 14(12):2120-2132. https:// doi.org/10.1016/j.jtho.2019.07.005

9. Engels M, Michael C, Dobra K, Hjerpe A, Fassina A, Firat $P$ (2019) Management of cytological material pre-analytical procedures and bio-banking in effusion cytopathology. Cytopathology 30:31-38

10. Fassunke J, Muller F, Keul M, Michels S, Dammert MA, Schmitt A, Plenker D, Lategahn J, Heydt C, Bragelmann J, Tumbrink HL, Alber $\mathrm{Y}$, Klein S, Heimsoeth A, Dahmen I, Fischer RN, Scheffler M, Ihle MA, Priesner V, Scheel AH, Wagener S, Kron A Frank K, Garbert K, Persigehl T, Pusken M, Haneder S, Schaaf B, Rodermann E, Engel-Riedel W, Felip E, Smit EF, Merkelbach-Bruse S, Reinhardt HC Kast SM, Wolf J, Rauh D, Buttner R, Sos ML (2018) Overcoming EGFR(G724S)-mediated osimertinib resistance through unique binding characteristics of second-generation EGFR inhibitors. Nat Commun 9:4655
11. Frampton GM, Ali SM, Rosenzweig M, Chmielecki J, Lu X, Bauer TM, Akimov M, Bufill JA, Lee C, Jentz D, Hoover R, Ou SH, Salgia R, Brennan T, Chalmers ZR, Jaeger $S$, Huang $A$, Elvin $J A$, Erlich R, Fichtenholtz A, Gowen KA, Greenbowe J, Johnson A, Khaira D, McMahon C, Sanford EM, Roels S, White J, Greshock J, Schlegel R, Lipson D, Yelensky R, Morosini D, Ross JS, Collisson E, Peters M, Stephens PJ, Miller VA (2015) Activation of MET via diverse exon 14 splicing alterations occurs in multiple tumor types and confers clinical sensitivity to MET inhibitors. Cancer Discov 5:850-859

12. Gainor JF, Dardaei L, Yoda S, Friboulet L, Leshchiner I, Katayama R, Dagogo-Jack I, Gadgeel S, Schultz K, Singh $M$, Chin E, Parks $M$, Lee $D$, DiCecca RH, Lockerman E, Huynh T, Logan J, Ritterhouse LL, Le LP, Muniappan A, Digumarthy S, Channick C, Keyes C, Getz G, Dias-Santagata D, Heist RS, Lennerz J, Sequist LV, Benes CH, lafrate AJ, Mino-Kenudson M, Engelman JA, Shaw AT (2016) Molecular mechanisms of resistance to first- and second-generation ALK inhibitors in ALK-rearranged lung cancer. Cancer Discov 6:1118-1133

13. Hagmeyer $L$, Fassunke J, Engels $M$, Treml $M$, Herkenrath S, Matthes S, Buttner R, Randerath W (2019) Bronchoscopic brushing from central lung cancer-next generation sequencing results are reliable. Lung 197:333-337

14. Hellmann MD, Ciuleanu TE, Pluzanski $A$, Lee JS, Otterson GA, Audigier-Valette $C$, Minenza $E$, Linardou $H$, Burgers $S$, Salman $P$, Borghaei $H_{\text {, }}$ Ramalingam SS, Brahmer J, Reck M, O'Byrne KJ, Geese WJ, Green G, Chang H, Szustakowski J, Bhagavatheeswaran P, Healey D, Fu Y, Nathan F, Paz-Ares L (2018) Nivolumab plus ipilimumab in lung cancer with a high tumor mutational burden NEngl J Med 378:2093-2104

15. Hellmann MD, Paz-Ares L, Bernabe Caro R, Zurawski $B$, Kim SW, Carcereny Costa E, Park K, Alexandru $A$, Lupinacci $L$, de la Mora Jimenez $E$ Sakai H, Albert I, Vergnenegre A, Peters S, Syrigos K, Barlesi F, Reck M, Borghaei H, Brahmer JR, O'Byrne KJ, Geese WJ, Bhagavatheeswaran P, Rabindran SK, Kasinathan RS, Nathan FE, Ramalingam SS (2019) Nivolumab plus ipilimumab in advanced non-small-cell lung cancer. N Engl J Med 381(21):2020-2031. https://doi.org/10.1056/ NEJMoa1910231

16. Herth FJ, Bubendorf L, Gutz S, Morresi-Hauf A Hummel M, Junker K, Lehmann U, Petersen I, Schnabel PA, Warth A (2013) Diagnostic and predictive analyses of cytological specimens of non-small cell lung cancer: strategies and challenges. Pneumologie 67:198-204

17. Hofman P, Heeke S, Alix-Panabieres C, Pantel K (2019) Liquid biopsy in the era of immuneoncology. Is it ready for prime-time use for cancer patients? Ann Oncol 30(9):1448-1459. https://doi. org/10.1093/annonc/mdz196

18. Hu Y, Ulrich BC, Supplee J, Kuang $Y$, Lizotte $\mathrm{PH}$ Feeney NB, Guibert NM, Awad MM, Wong KK, Janne PA, Paweletz CP, Oxnard GR (2018) Falsepositive plasma genotyping due to clonal hematopoiesis. Clin Cancer Res 24:4437-4443

19. Ilie M, Beaulande M, Hamila M, Erb G, Hofman V, Hofman P (2018) Automated chromogenic multiplexed immunohistochemistry assay for diagnosis and predictive biomarker testing in nonsmall cell lung cancer. Cancer Treat Res 124:90-94

20. Junker K, Buttner R, Langer T, Ukena D (2018) Pathological-anatomical diagnosis according to the German lung cancer guideline 2018. Pathologe 39:589-603 
21. Kron A, Alidousty C, Scheffler M, MerkelbachBruse $S$, Seidel D, Riedel R, Ihle MA, Michels $S$, Nogova L, Fassunke J, Heydt C, Kron F, Ueckeroth F, Serke M, Kruger S, Grohe C, Koschel D, Benedikter J, Kaminsky B, Schaaf B, Braess J, Sebastian M, Kambartel KO, Thomas R, Zander T, Schultheis AM, Buttner R, Wolf J (2018) Impact of TP53 mutation status on systemic treatment outcome in ALKrearranged non-small-cell lung cancer. Ann Oncol 29:2068-2075

22. Leighl NB, Page RD, Raymond VM, Daniel DB, Divers SG, Reckamp KL, Villalona-Calero MA, Dix D Odegaard JI, Lanman RB, Papadimitrakopoulou VA (2019) Clinical utility of comprehensive cell-free DNA analysis to identify genomic biomarkers in patients with newly diagnosed metastatic non-small cell lung cancer. Clin Cancer Res 25:4691-4700

23. Meder L, Konig K, Fassunke J, Ozretic L, Wolf J, Merkelbach-Bruse S, Heukamp LC, Buettner R (2015) Implementing amplicon-based next generation sequencing in the diagnosis of small cell lung carcinoma metastases. Exp Mol Pathol 99:682-686

24. Michels S, Massuti B, Schildhaus HU, Franklin J, Sebastian M, Felip E, Grohe C, RodriguezAbreu D, Abdulla DSY, Bischoff $H$, Brandts C, Carcereny E, Corral J, Dingemans AC, Pereira E, Fassunke J, Fischer RN, Gardizi M, Heukamp L, Insa A, Kron A, Menon R, Persigehl T, Reck M, Riedel R, Rothschild SI, Scheel AH, Scheffler M, Schmalz P, Smit EF, Limburg M, Provencio M, Karachaliou N, Merkelbach-Bruse S, Hellmich M, Nogova L, Buttner R, Rosell R, Wolf J (2019) Safety and efficacy of crizotinib in patients with advanced or metastatic ROS1-rearranged lung cancer (EUCROSS): a European phase II clinical trial. JThorac Oncol 14:1266-1276

25. Peters S, Camidge DR, Shaw AT, Gadgeel S, Ahn JS, Kim DW, Ou SI, Perol M, Dziadziuszko R, Rosell R, Zeaiter A, Mitry E, Golding S, Balas B, Noe J, Morcos PN, Mok T, Investigators AT (2017) Alectinib versus crizotinib in untreated ALK-positive nonsmall-cell lung cancer. N Engl J Med 377:829-838

26. Plenker $D$, Bertrand $M$, de Langen $A J$, Riedel $R$ Lorenz C, Scheel AH, Muller J, Bragelmann J, Dassler-Plenker J, Kobe C, Persigehl T, Kluge A, Wurdinger T, Schellen P, Hartmann G, Zacherle T, Menon R, Thunnissen E, Buttner R, Griesinger F, Wolf J, Heukamp L, Sos ML, Heuckmann JM (2018) Structural alterations of MET trigger response to MET Kinase inhibition in lung adenocarcinoma patients. Clin Cancer Res 24:1337-1343

27. Reck $M$, Schenker $M$, Lee $K H$, Provencio $M$, Nishio M, Lesniewski-Kmak K, Sangha R, Ahmed S, Raimbourg J, Feeney K, Corre R, Franke FA, Richardet E, Penrod JR, Yuan Y, Nathan FE, Bhagavatheeswaran P, DeRosa M, Taylor F, Lawrance R, Brahmer J (2019) Nivolumab plus ipilimumab versus chemotherapy as first-line treatment in advanced non-small-cell lung cancer with high tumour mutational burden: patientreported outcomes results from the randomised, open-label, phase III CheckMate 227 trial. Eur J Cancer 116:137-147

28. Schildhaus HU, Schultheis AM, Ruschoff J, Binot E, Merkelbach-Bruse S, Fassunke J, Schulte W, Ko YD, Schlesinger A, Bos M, Gardizi M, EngelRiedel W, Brockmann $M$, Serke $M$, Gerigk U, Hekmat K, Frank KF, Reiser M, Schulz H, Kruger S, Stoelben E, Zander T, Wolf J, Buettner R (2015) MET amplification status in therapy-naive adeno- and squamous cell carcinomas of the lung. Clin Cancer Res 21:907-915
29. Soria JC, Ohe Y, Vansteenkiste J, Reungwetwattana T, Chewaskulyong B, Lee KH, Dechaphunkul A, Imamura F, Nogami N, Kurata T, Okamoto I, Zhou C, Cho BC, Cheng Y, Cho EK, Voon PJ, Planchard D, Su WC, Gray JE, Lee SM, Hodge R, Marotti M, Rukazenkov $Y$, Ramalingam SS, Investigators $F$ (2018) Osimertinib in untreated EGFR-mutated advanced non-small-cell lung cancer. N Engl J Med 378:113-125

30. Soria JC, Ramalingam SS (2018) Osimertinib in EGFR mutation-positive advanced NSCLC. N Engl J Med 378:1262-1263

31. Thunnissen $E$, Borczuk AC, Flieder DB, Witte $B$, Beasley MB, Chung JH, Dacic S, Lantuejoul $S$, Russell PA, den Bakker M, Botling J, Brambilla E, de Cuba E, Geisinger KR, Hiroshima K, Marchevsky AM, Minami Y, Moreira A, Nicholson AG, Yoshida A, Tsao MS, Warth A, Duhig E, Chen G, Matsuno Y, Travis WD, Butnor K, Cooper W, Mino-Kenudson M Motoi N, Poleri C, Pelosi G, Kerr K, Aisner SC, Ishikawa Y, Buettner RH, Keino N, Yatabe Y, Noguchi $M$ (2017) The use of immunohistochemistry improves the diagnosis of small cell lung cancer and its differential diagnosis. An international reproducibility study in a demanding set of cases. JThorac Oncol 12:334-346

32. Travis WD, Brambilla $E$, Nicholson AG, Yatabe $Y$, Austin JHM, Beasley MB, Chirieac LR, Dacic $S$, Duhig E, Flieder DB, Geisinger K, Hirsch FR, Ishikawa Y, Kerr KM, Noguchi M, Pelosi G, PowellCA, Tsao MS, Wistuba I, WHO Panel (2015) The 2015 world health organization classification of lung tumors: impact of genetic, clinical and radiologic advances since the 2004 classification. J Thorac Oncol 10:1243-1260

33. Vassella $E$, Langsch $S$, Dettmer MS, Schlup $C$, Neuenschwander M, Frattini M, Gugger M, Schafer SC (2015) Molecular profiling of lung adenosquamous carcinoma: hybrid or genuine type? Oncotarget 6:23905-23916

34. Yatabe Y, Dacic S, Borczuk AC, Warth A, Russell PA, Lantuejoul S, Beasley MB, Thunnissen E, Pelosi G, Rekhtman N, Bubendorf L, Mino-Kenudson M, Yoshida A, Geisinger KR, Noguchi M, Chirieac LR, Bolting J, Chung JH, Chou TY, Chen G, Poleri C, Lopez-Rios F, Papotti M, Sholl LM, Roden AC, Travis WD, Hirsch FR, Kerr KM, Tsao MS, Nicholson AG, Wistuba I, Moreira AL (2019) Best practices recommendations for diagnostic immunohistochemistry in lung cancer. JThorac Oncol 14:377-407
Lichttherapie für Immunzellen hilft bei Nebenwirkungen von Krebstherapie

Eine häufige Nebenwirkung von Krebs-Immuntherapien lässt sich vermutlich durch Lichtaktivierung von Immunzellen stoppen, wie Forscher des Universitätsklinikums Freiburg zeigen

Erst seit wenigen Jahren ist schwarzer Hautkrebs, auch Melanom genannt, medikamentös behandelbar. Allerdings kommt es bei jedem zweiten Patienten zu starken Autoimmunreaktionen wie Hautausschlag oder Durchfall. Dass sich diese mit einer speziellen Lichttherapie aufhalten lassen, haben jetzt Forscherinnen und Forscher des Universitätsklinikums Freiburg mit schweizerischen Kollegen gezeigt. Einen 29-jährigen Krebspatienten mit einer schweren Entzündung der Darmschleimhaut behandelten sie sehr erfolgreich mittels Extrakorporaler Photopherese (ECP). Dabei werden Immunzellen außerhalb des Körpers mit einem lichtreaktiven Medikament versetzt, mit UV-Licht bestrahlt und in den Körper zurückgegeben. Durch das Verfahren werden vermutlich Immunzellen aktiviert, die die Entzündung stoppen. Die Photopherese-Therapie führte bei dem Patienten zu einem vollständigen Abklingen der Beschwerden, während durch die fortlaufende Krebstherapie der Hautkrebs erfolgreich behandelt werden konnte. Bisherige, etablierte Behandlungsansätze waren im Vorfeld erfolglos geblieben. Die Fallbeschreibung erschien am 15. Januar 2020 im renommierten Fachmagazin New England Journal of Medicine.

Literatur: Extracorporeal Photopheresis for Colitis Induced by Checkpoint-Inhibitor Therapy DOI: 10.1056/NEJMc1912274

Quelle: Universitätsklinikum Freiburg, 16.01.2020 